\title{
Estimating solar radiation using NOAA/AVHRR and ground measurement data
}

\begin{abstract}
Solar radiation (SR) data are commonly used in different areas of renewable energy research. Researchers are often compelled to predict SR at ground stations for areas with no proper equipment. The objective of this study was to test the accuracy of the artificial neural network (ANN) and multiple linear regression (MLR) models for estimating monthly average SR over Kurdistan Province, Iran. Input data of the models were two data series with similar longitude, latitude, altitude, and month (number of months) data, but there were differences between the monthly mean temperatures in the first data series obtained from AVHRR sensor of NOAA satellite (DS1) and in the second data series measured at ground stations (DS2). In order to retrieve land surface temperature (LST) from AVHRR sensor, emissivity of the area was considered and for that purpose normalized vegetation difference index (NDVI) calculated from channels 1 and 2 of AVHRR sensor was utilized. The acquired results showed that the ANN model with DS1 data input with $\mathrm{R} 2=0.96, \mathrm{RMSE}=1.04, \mathrm{MAE}=1.1$ in the training phase and $\mathrm{R} 2=0.96, \mathrm{RMSE}=1.06, \mathrm{MAE}=1.15$ in the testing phase achieved more satisfactory performance compared with MLR model. It can be concluded that ANN model with remote sensing data has the potential to predict SR in locations with no ground measurement stations.
\end{abstract}

Keyword: AVHRR sensor; Land surface temperature; NDVI; Emissivity 